\title{
Conocimiento y percepción de la eutanasia en estudiantes y profesionales de medicina
}

\section{Medical students and physicians' knowledge and perceptions about euthanasia}

\author{
Rosolina D'Amico López, ${ }^{*}$ Ginno Alessandro \\ de Benedictis Serrano, ${ }^{* *}$ Hadvy Humberto Coiran \\ Mendoza, ${ }^{* * *}$ Joaquín Fernando Castro Álvarez ${ }^{* * * *}$
}

https://doi.org/10.36105/mye.2020v31n3.05

Autofinanciado por los autores.

Los autores no declaran ningún conflicto de interés.

* Facultad de Ciencias de la Salud, Universidad de Carabobo, Sede Aragua. Venezuela. https://orcid.org/0000-0001-7991-0389

** Facultad de Ciencias de la Salud, Universidad de Carabobo, Sede Aragua. Venezuela. Correo electrónico: sparck864@gmail.com https://orcid.org/00000002-3191-7167

*** Facultad de Ciencias de la Salud, Universidad de Carabobo, Sede Aragua. Venezuela. https://orcid.org/0000-0002-9463-2601

**** Facultad de Ciencias de la Salud, Universidad de Carabobo, Sede Aragua. Venezuela. https://orcid.org/0000-0002-1423-2086

Recepción: 20 de marzo de 2020. Aceptación: 15 de mayo de 2020. 


\section{Resumen}

Antecedente. Se considera "eutanasia» al acto de acabar con la vida del paciente a petición expresa de él, para terminar el sufrimiento propio de alguna enfermedad sin perspectiva de cura.

Objetivo. Evaluar los conocimientos y la percepción sobre la eutanasia en estudiantes y profesionales de medicina, demostrando los conocimientos y clasificando la percepción sobre la eutanasia.

Metodología. Estudio clínico-epidemiológico, observacional, cualitativo-cuantitativo, de corte transversal y con muestreo no probabilístico por conveniencia, en los estudiantes y profesionales de medicina de un hospital en Venezuela.

Resultados. La muestra estudiada tuvo una distribución según grado de instrucción: $64.85 \%$ de estudiantes, $19.33 \%$ de residentes y $15.82 \%$ de especialistas. Presentaron un grado de conocimiento medio, distribuido en $84.4 \%$ de especialistas, $77.3 \%$ de residentes y $80.5 \%$ de estudiantes (p 0.051). De acuerdo con los grados de conocimiento y el apoyo a la eutanasia, el $76.18 \%$ tiene un grado medio de conocimientos ( $p$ 0.0002).

Conclusión. Se demostró que existe un grado medio de conocimiento, en particular en la población estudiantil. La eutanasia no fue percibida de manera negativa, demostrando una alta significancia en la atención a las implicaciones éticas que rodean este concepto.

Palabras clave: bioética, derecho a morir, eutanasia, actitud frente a la muerte.

\section{Introducción}

La eutanasia ha sido y es un tema controvertido en la sociedad, principalmente entre los profesionales de la salud. Entre las definiciones del término, la más básica lo conceptualiza como «el acto de acabar con la vida del paciente a petición expresa de él, para terminar el sufrimiento propio de alguna enfermedad sin perspectiva de cura». ${ }^{1,2}$ Se distingue del suicidio asistido, en donde el médico se 
limita a proporcionar a la persona los medios para que se suicide, pero no realiza la acción que causa la muerte. ${ }^{3}$

Existen otros enfoques sobre la muerte en pacientes con enfermedades terminales, como es la «distanasia», que se define como «la prolongación artificial de la vida biológica de un paciente con enfermedad irreversible o terminal por medio de la tecnología médica». ${ }^{4}$ En contraparte, existe la «ortotanasia», la cual se considera como la buena muerte, en el sentido de muerte en el momento biológico adecuado, lo que se infiere como una actuación correcta ante la muerte por parte de los cuidadores de pacientes con enfermedades terminales, caso que se observa principalmente en la medicina paliativa. Hay que tener en cuenta que las dos corrientes antes mencionadas se enfocan en preservar la vida.

En la actualidad se puede apreciar una alta productividad científica sobre la eutanasia, un tema controvertido a nivel mundial. Se tiende a dividir en activa y pasiva, siendo la primera la acción que realiza un médico para producir la muerte de un paciente, sin dolor y a petición de éste, la cual es legal en Holanda, Bélgica, Luxemburgo y en Colombia. Mientras tanto, la pasiva es cuando se deja de dar o se retira un tratamiento con la consecuente muerte del paciente, permitida con diversas regulaciones en Canadá, España, Inglaterra, India, entre otros, siendo ésta una práctica ampliamente usada en medicina intensiva a nivel mundial. ${ }^{5}$

La eutanasia ha sido practicada desde los inicios de la medicina y ha generado discusiones presentes hasta la actualidad, principalmente condenada por muchas religiones. ${ }^{6}$ Desde el enfoque de la bioética existen confrontaciones ya que, por un lado, toda persona tiene el derecho a decidir el tratamiento que ha de recibir u omitir, lo que se considera como el principio de autonomía, pero, por otro lado, al atentar contra la vida, se transgrede el principio de la no maleficencia ${ }^{7}$ y la supremacía del derecho a la vida, ratificada en la Declaración Universal de Derechos Humanos por la Organización de Naciones Unidas (ONU). ${ }^{8}$ 
En este estricto sentido, es conveniente destacar el Código de Deontología Médica de Venezuela aprobado en 2004, que en su capítulo quinto contiene los siguientes artículos:?

Artículo 79.- Los objetivos fundamentales en el tratamiento de un paciente terminal son el alivio del sufrimiento, propiciar la mayor comodidad posible, facilitar el contacto con los seres queridos, recibir la ayuda espiritual del ministro o sacerdote de su religión si la tiene y si así lo desea y, finalmente, ayudarlo a enfrentar la muerte con dignidad.

Artículo 82.- El enfermo terminal no debe ser sometido a la aplicación de medidas de soporte vital derivadas de la tecnología, las cuales sólo servirán para prolongar la agonía y no para preservar la vida.

Parágrafo Uno. Distanasia: es la utilización de medidas terapéuticas de soporte vital que llevan a posponer la muerte en forma artificial. Es equivalente a ensañamiento terapéutico, encarnizamiento terapéutico o hiperterapéutico.

Parágrafo Dos. En aquel paciente críticamente enfermo, cuya evaluación por consenso de los médicos tratantes sea considerado como enfermo terminal, la conducta se regirá por lo contemplado en este artículo.

Artículo 84.- Es obligación fundamental del médico el alivio del sufrimiento humano. No puede, en ninguna circunstancia, provocar deliberadamente la muerte del enfermo, aun cuando éste o sus familiares lo soliciten, como tampoco deberá colaborar o asistir al suicidio del paciente instruyéndolo y/o procurándole un medicamento en una dosis letal.

La discusión sobre la eutanasia, su legislación y su moralidad, es un tópico constante y presente en todos los tiempos, sólo que es evitado o simplemente prohibido, ${ }^{10}$ e incluso ignorado. Sin embargo, hoy es un tema muy debatido en los medios de comunicación y por la población en general. Parece que hay una falta de claridad en los conceptos y definiciones utilizados en el debate social de la eutanasia, lo que conlleva a que las discusiones sobre el tema sean a menudo confusas. 
En España, en el año 2015, se realizó una investigación cualitativa en grupos de médicos que atienden pacientes en situación terminal, buscando principalmente la definición correcta de la eutanasia, mostrándose la falta de consenso en relación con dicho concepto. En el mismo año, en Perú, se realizó una investigación en médicos internos y residentes de un hospital, y se encontró que el $61 \%$ de los internos presentaba un buen nivel de conocimiento sobre la eutanasia, y que el $88 \%$ presentaba una actitud negativa hacia esta práctica. ${ }^{11}$

En Alemania, en el 2016, se demostró que el 19\% de los estudiantes de medicina de una universidad consideraron la eutanasia como éticamente permisible, y mostraron interés en profundizar acerca de los elementos éticos en la carrera. ${ }^{12}$ En el año 2018 se hizo un estudio bibliométrico, el cual arrojó una alta producción científica sobre la eutanasia, lo que denota el gran estudio en torno a este tema; sin embargo, no se han establecido las bases a nivel mundial sobre el tópico.

Para comprender el pensamiento existente sobre la eutanasia, se deben entender los conceptos de conocimiento y percepción. Actualmente no existe un acuerdo universal en lo que respecta al conocimiento verdadero sobre algo, por lo que se puede definir el conocer como el proceso de descifrar por medio de las aptitudes intelectuales, la naturaleza, características y relaciones de las cosas. El conocimiento puede variar, desde el científico hasta el empírico, y esto está subordinado al grado de los nexos que se instauren entre los elementos que configuran el proceso de conocimiento. ${ }^{13}$

En contraste con lo anteriormente expresado, la percepción se define, de manera tradicional, como un proceso cognitivo originario de la conciencia que radica en el reconocimiento, en la interpretación y significación para la construcción de juicios en torno a las sensaciones adquiridas en el ambiente físico y social, interviniendo procesos de asimilación como el aprendizaje, y psíquicos tales como la memoria y la simbolización. ${ }^{14}$ Principalmente, la percepción precisa el recibimiento de información desde el ambiente, 
consciente de que la misma es utilizada para elaborar abstracciones. $^{15}$

Estimar el conocimiento de estudiantes y profesionales de medicina referente a la eutanasia, previo a indagar la percepción que puedan tener tan distintas poblaciones, no es algo que se haya realizado con anterioridad. Sin embargo, existen estudios que se relacionan con la búsqueda sobre lo que piensan las personas acerca de la eutanasia; no obstante, son pocos los trabajos que combinan las opiniones de estudiantes y profesionales de medicina. Precisamente por todo lo anterior, se planteó como objetivo evaluar los conocimientos y la percepción sobre la eutanasia en estudiantes y profesionales de medicina en el Hospital Central de Maracay, describiendo las características sociodemográficas, demostrando los conocimientos y clasificando la percepción sobre la eutanasia en los estudiantes y profesionales de medicina.

\section{Materiales y métodos}

Se llevó a cabo un estudio clínico-epidemiológico, con modalidad observacional, además de cualitativo-cuantitativo, de corte transversal y con muestreo no probabilístico por conveniencia, en los estudiantes y profesionales de medicina del Hospital Central de Maracay, durante los meses de mayo a agosto del 2019.

Fueron incluidos 369 estudiantes de cuarto a sexto año de la carrera de medicina de las universidades integradas al Hospital Central de Maracay, y 200 profesionales de medicina del mismo, los cuales accedieron a participar en el estudio. No se incluyó en la investigación a quien decidió no participar en la misma y a aquellos que notificaron no tener conocimientos sobre eutanasia.

Previo a la ejecución del estudio, se obtuvo la aprobación por parte de la dirección del Hospital Central de Maracay. Respetando las normas éticas contenidas en el Código de Ética para la Vida del Ministerio del Poder Popular para la Ciencia, Tecnología e Indus- 
trias Intermedias de la República Bolivariana de Venezuela, y en concordancia con los principios éticos de no maleficencia, beneficencia, autonomía y precaución, expuestos en la Declaración de Helsinki, se solicitó a los participantes el consentimiento informado de participación en la investigación.

Para la ejecución de este trabajo se elaboró un instrumento de recolección de datos, el cual constó de 3 partes y de 29 preguntas cerradas, el cual fue validado por el juicio de expertos. Fue aplicado bajo la técnica de la encuesta. En la primera parte se encuentran los datos sociodemográficos, que constan de 5 preguntas sobre edad, sexo, grado de instrucción, religión y estado civil. La segunda parte consta de 10 preguntas, que incluyen todas las nociones básicas para considerar si los participantes tienen conocimientos generales sobre todo lo referente a la eutanasia. La tercera parte se encuentra conformada por 14 preguntas que indagan sobre la percepción u opinión de los participantes en distintas situaciones o temas referentes a la eutanasia. Posteriormente, se procedió a categorizar en grados de conocimiento las respuestas correctas, de acuerdo con la mediana y el percentil 25 y 75, y se estableció que aquellos con una puntación de 0 a 3 tienen un grado bajo de conocimiento; de 4 a 7, un grado medio, y de 8 a 10, un grado alto. Entre las limitaciones observadas en la investigación se tienen en cuenta la falta de tiempo por parte de los profesionales de medicina para llenar un instrumento a través de una encuesta, y la ausencia de áreas comunes de esparcimiento dentro del Hospital Central de Maracay.

La presentación de los datos se realizó a través de tablas. Para la estadística descriptiva se emplearon distribuciones de frecuencias para las variables cualitativas, y promedio, desviación estándar y mediana para las variables cuantitativas. Para la estadística analítica se construyeron tablas de asociación mediante la prueba Chi-Cuadrado, con un nivel de significancia $\mathrm{p} \leq 0.05$. Para la realización de estos análisis se empleó el programa EpiInfo 7.2, vaciándose los datos previamente tabulados en una hoja de cálculo de Excel. 


\section{Resultados}

En el estudio fueron incluidos 369 estudiantes de la carrera de medicina y 200 profesionales de medicina. El rango de edad en el que se concentraron más encuestados fue de 20 a 25 años, con un $63.09 \%$;

Tabla 1. Distribución de los estudiantes y profesionales de medicina,

de acuerdo con características generales y grado de conocimiento.

\begin{tabular}{|c|c|c|c|}
\hline Variable & $\mathbf{n}$ & $\%$ & IC $95 \%$ \\
\hline \multicolumn{4}{|l|}{ Edad } \\
\hline 21 a 30 años & 473 & 83.13 & $79.83-85.98$ \\
\hline 31 a 40 años & 62 & 10.90 & $8.59-13.72$ \\
\hline 41 a 50 años & 13 & 2.28 & $1.34-3.87$ \\
\hline 51 a 60 años & 18 & 3.16 & $2.01-4.94$ \\
\hline 61 o más años & 3 & 0.53 & $0.18-1.54$ \\
\hline \multicolumn{4}{|l|}{ Sexo } \\
\hline Femenino & 335 & 58.88 & $54.79-62.85$ \\
\hline Masculino & 234 & 41.12 & $37.15-45.21$ \\
\hline \multicolumn{4}{|l|}{ Grado de instrucción } \\
\hline Especialistas & 90 & 15.82 & $13.05-19.04$ \\
\hline Residentes & 110 & 19.33 & $60.84-68.66$ \\
\hline Estudiantes & 369 & 64.85 & $16.30-22.78$ \\
\hline \multicolumn{4}{|l|}{ Religión } \\
\hline Cristiana católica & 346 & 60.81 & $56.74-64.73$ \\
\hline Cristiana evangélica & 105 & 18.45 & $15.48-21.85$ \\
\hline Atea & 57 & 10.02 & $7.81-12.76$ \\
\hline Agnóstica & 39 & 6.85 & $5.05-9.23$ \\
\hline Deísta & 10 & 1.76 & $0.96-3.20$ \\
\hline Adventista & 8 & 1.41 & $0.71-2.75$ \\
\hline Testigo de Jehová & 3 & 0.53 & $0.18-1.54$ \\
\hline Judía & 1 & 0.18 & $0.03-0.99$ \\
\hline \multicolumn{4}{|l|}{ Estado civil } \\
\hline Soltero/a & 487 & 85.59 & $82.46-88.24$ \\
\hline Casado/a & 57 & 10.02 & $7.81-12.76$ \\
\hline Unión libre & 21 & 3.69 & $2.43-5.58$ \\
\hline Separado/a & 3 & 0.53 & $0.18-1.54$ \\
\hline Viudo/a & 1 & 0.18 & $0.03-0.99$ \\
\hline \multicolumn{4}{|c|}{ Grado de conocimientos de la eutanasia } \\
\hline Alto & 70 & 12.30 & $9.85-15.26$ \\
\hline Medio & 458 & 80.49 & $77.04-83.54$ \\
\hline Bajo & 41 & 7.21 & $5.36-9.63$ \\
\hline
\end{tabular}


335 participantes fueron de sexo femenino, mientras que 234 fueron de sexo masculino. La población de estudiantes fue de $64.85 \%$, mientras que la de residentes fue de $19.33 \%$, y la de especialistas, 15.82\%. La religión cristiana católica predominó con el $60.81 \%$, seguida de la religión cristiana evangélica con el 18.45\%. El $85.59 \%$ de los participantes eran solteros. El 80.49\% posee un grado de conocimientos medio con respecto a la eutanasia (Tabla 1).

Con respecto a la percepción, el $38.14 \%$ está en desacuerdo en considerar la eutanasia como un homicidio; el $37.26 \%$ totalmente en desacuerdo en ver la eutanasia como pecado; el 39.54\% totalmente en desacuerdo en percibir la eutanasia como un acto inmoral, mientras que el 36.81\% considera la eutanasia como una muerte digna. El 37.96\% de los participantes acepta la eutanasia en pacientes en situaciones críticas, mientras que el 45.34\% está totalmente en desacuerdo con respecto a la aplicación de medidas que provocan directamente la muerte, sin importar el padecimiento. El $38.14 \%$ está en desacuerdo con respecto a percibir la eutanasia como desvalorización de la profesión médica. Un 30.58\% de los participantes está de acuerdo con preservar la vida hasta su fin natural, pero el $42.00 \%$ considera que el sufrimiento es importante para la toma de decisión de realizar la eutanasia. El 35.15\% está de acuerdo con morir si se va a vivir sin una calidad de vida adecuada (Tabla 2 de la página siguiente).

Se observó que, de acuerdo con el grado de instrucción, la mayoría presentó un nivel de conocimiento medio (especialistas $84.4 \%$, residentes $77.3 \%$ y estudiantes $80.5 \%$ ), p 0.051 (Tabla 3). Las respuestas afirmativas con respecto a los participantes que consideran ética la eutanasia presentaban, en su mayoría, niveles altos $(14.21 \%)$ y medios $(76.50 \%)$ de conocimiento sobre la misma, p 0.0029 (Tabla 4).

Cabe destacar que, entre los que respondieron afirmativamente en apoyo a la eutanasia, se representó según sexo en $56.02 \%$ femenino ( $\mathrm{p}$ 0.03); en este mismo grupo se observó que, de acuerdo con el grado de instrucción de los estudiantes de medicina repre- 
R. D’Amico López, G. Benedictis Serrano, H. Coiran Mendoza, J. Castro Álvarez

Tabla 2. Percepción de los estudiantes y profesionales de medicina ante diversos contextos referentes a la eutanasia.

\begin{tabular}{|c|c|c|c|c|c|}
\hline Variable & $\begin{array}{c}\text { Totalmente } \\
\text { en desacuerdo } \\
n(\%)\end{array}$ & $\begin{array}{c}\text { En } \\
\text { desacuerdo } \\
n(\%)\end{array}$ & $\begin{array}{c}\text { Indeciso/ } \\
\text { dudoso } \\
\text { n (\%) }\end{array}$ & $\begin{array}{c}\text { De } \\
\text { acuerdo } \\
\text { n (\%) }\end{array}$ & $\begin{array}{c}\text { Totalmente } \\
\text { de acuerdo } \\
\text { n (\%) }\end{array}$ \\
\hline Eutanasia cc & $\begin{array}{l}\text { «homicidio» } \\
192(33.74)\end{array}$ & $217(38.14)$ & $115(20.21)$ & $26(4.57)$ & $19(3.34)$ \\
\hline Eutanasia cc & $\begin{array}{l}\text { «pecado» } \\
212(37.26)\end{array}$ & $152(26.71)$ & 109(19.16) & $76(13.36)$ & $20(3.51)$ \\
\hline Eutanasia cc & $\begin{array}{l}\text { «inmoral» } \\
225(39.54)\end{array}$ & $185(32.51)$ & 111(19.51) & $30(5.27)$ & 18(3.16) \\
\hline Eutanasia cc & $\begin{array}{c}\text { «muerte digna» } \\
52(9.20)\end{array}$ & $73(12.92)$ & $130(23.01)$ & $208(36.81)$ & $102(18.05)$ \\
\hline Aceptación & $\begin{array}{l}\text { eutanasia en pac } \\
37(6.50)\end{array}$ & $\begin{array}{c}\text { cientes en situ } \\
46(8.08)\end{array}$ & $\begin{array}{l}\text { aciones crítice } \\
97(17.05)\end{array}$ & $216(37.96)$ & $173(30.40)$ \\
\hline Aplicación d & $\begin{array}{c}\text { didas que provoq } \\
258(45.34)\end{array}$ & $\begin{array}{c}\text { quen la muerte } \\
196(34.45)\end{array}$ & $\begin{array}{c}\text { sin importar } \\
62(10.90)\end{array}$ & $\begin{array}{l}\text { padecimie } \\
36(6.33)\end{array}$ & $\begin{array}{l}\text { to } \\
17(2.99)\end{array}$ \\
\hline Eutanasia cc & $\begin{array}{c}\text { desvalorización de } \\
179(31.46)\end{array}$ & $\begin{array}{l}\text { le la profesión } \\
217(38.14)\end{array}$ & $\begin{array}{l}\text { médica } \\
100(17.57)\end{array}$ & $44(7.73)$ & $29(5.10)$ \\
\hline Preservar la & $\begin{array}{l}\text { hasta su fin natur } \\
47(8.26)\end{array}$ & $\begin{array}{l}\text { ural } \\
\qquad 89(15.64)\end{array}$ & $157(27.59)$ & $174(30.58)$ & $102(17.93)$ \\
\hline Sufrimiento & $\begin{array}{l}\text { o factor importante } \\
53(9.31)\end{array}$ & $\begin{array}{c}\text { e para realizar } \\
33(5.80)\end{array}$ & $\begin{array}{c}\text { la eutanasia } \\
52(9.14)\end{array}$ & $239(42.00)$ & 192(33.74) \\
\hline Preferencia & $\begin{array}{c}\text { norir a vivir sin cali } \\
54(9.49)\end{array}$ & $\begin{array}{c}\text { lidad de vida a } \\
91(15.99)\end{array}$ & $\begin{array}{l}\text { decuada } \\
151(26.54)\end{array}$ & $200(35.15)$ & 73(12.83) \\
\hline
\end{tabular}

sentan el $65.71 \%$; los especialistas ocuparon un $18.32 \%$, y los residentes $15.97 \%$ ( $\mathrm{p}$ 0.0013). De los participantes que apoyan afirmativamente la eutanasia, el $82.98 \%$ ha estado en contacto con pacientes en estado terminal ( $p$ 0.0009), mientras que, de acuerdo con los grados de conocimiento y el apoyo a la eutanasia, el $13.87 \%$ tiene un grado alto de conocimiento y el $76.18 \%$ tiene un grado medio de conocimientos (0.0002) (Tabla 5). 
Conocimiento de la eutanasia en estudiantes y profesionales de medicina

Tabla 3. Relación entre el nivel de conocimiento y el grado de instrucción.

\begin{tabular}{|c|c|c|c|c|}
\hline \multicolumn{5}{|c|}{ Grado de instrucción } \\
\hline Variable & $\begin{array}{c}\text { Especialista } \\
(n=90) \\
n(\%)\end{array}$ & $\begin{array}{c}\text { Residente } \\
(n=110) \\
n(\%)\end{array}$ & $\begin{array}{c}\text { Estudiante } \\
\text { (n= 369) } \\
n(\%)\end{array}$ & $\mathbf{P}$ \\
\hline \multicolumn{5}{|c|}{ Grado de conocimiento de la eutanasia } \\
\hline Alto & $6(6.7)$ & 21(19.1) & $43(11.7)$ & \\
\hline Medio & $76(84.4)$ & $85(77.3)$ & $297(80.5)$ & 0.051 \\
\hline Bajo & 8(8.9) & $4(3.6)$ & $29(7.9)$ & \\
\hline
\end{tabular}

Tabla 4. Relación entre el grado de conocimiento y la consideración ética de la eutanasia.

\begin{tabular}{|c|c|c|c|c|}
\hline \multirow[b]{2}{*}{ Variable } & \multicolumn{3}{|c|}{ Consideración ética de la eutanasia } & \multirow[t]{2}{*}{$\mathbf{P}$} \\
\hline & $\begin{array}{c}\text { Sí (n = 366) } \\
n(\%)\end{array}$ & $\begin{array}{c}\text { No }(n=110) \\
n(\%)\end{array}$ & $\begin{array}{c}\text { No sé }(n=93) \\
n(\%)\end{array}$ & \\
\hline \multicolumn{5}{|c|}{ Grado de conocimiento de la eutanasia } \\
\hline Alto & $52(14.21)$ & $12(10.91)$ & $6(6.45)$ & \\
\hline Medio & $280(76.50)$ & $98(89.09)$ & $7(7.53)$ & 0.0029 \\
\hline Bajo & $34(9.29)$ & - & $80(86.02)$ & \\
\hline
\end{tabular}

Tabla 5. Relación entre características generales y apoyo a la eutanasia.

\begin{tabular}{|c|c|c|c|c|c|}
\hline \multirow{2}{*}{\multicolumn{2}{|c|}{ Variable }} & \multicolumn{3}{|c|}{ Apoyo a la eutanasia } & \multirow[b]{2}{*}{$\mathbf{P}$} \\
\hline & & $\begin{array}{c}\text { No }(n=101) \\
n(\%)\end{array}$ & $\begin{array}{c}\text { No sé }(n=85) \\
n(\%)\end{array}$ & $\begin{array}{c}\text { Sí (n = 382) } \\
n(\%)\end{array}$ & \\
\hline \multirow[t]{2}{*}{ Sexo } & Femenino & $60(59.41)$ & $61(70.93)$ & $214(56.02)$ & \multirow[t]{2}{*}{0.03} \\
\hline & Masculino & $41(40.59)$ & $25(29.07)$ & 168(43.98) & \\
\hline \multicolumn{6}{|c|}{ Grado de instrucción } \\
\hline & Especialistas & $11(10.89)$ & $9(10.47)$ & $70(18.32)$ & \multirow{3}{*}{0.0013} \\
\hline & Residentes & $33(32.67)$ & $16(18.60)$ & $61(15.97)$ & \\
\hline & Estudiantes & $57(56.44)$ & $61(70.93)$ & $251(65.71)$ & \\
\hline \multicolumn{6}{|c|}{ Contacto con pacientes en estado terminal } \\
\hline & Sí & $78(77.23)$ & $56(65.12)$ & $317(82.98)$ & \multirow[t]{2}{*}{0.0009} \\
\hline & No & $23(22.77)$ & $30(34.88)$ & $65(17.02)$ & \\
\hline \multicolumn{6}{|c|}{ Grado de conocimientos } \\
\hline & Alto & $14(13.86)$ & $3(3.49)$ & $53(13.87)$ & \multirow{3}{*}{0.0002} \\
\hline & Medio & $87(86.14)$ & $80(93.02)$ & $291(76.18)$ & \\
\hline & Bajo & $0(0.00)$ & $3(3.49)$ & $38(9.95)$ & \\
\hline
\end{tabular}




\section{Discusión}

La muestra recogida estuvo conformada en un tercio por profesionales, y dos tercios por estudiantes de medicina, lo que demuestra un estudio más heterogéneo, en contraste con una investigación cuya población estuvo netamente conformada por estudiantes de medicina, como el de Ríos González et al. en 2018, que fue aplicada a estudiantes de medicina de Latinoamérica. ${ }^{16}$ En el presente estudio, más de tres cuartas partes de los participantes poseía un grado medio de conocimientos sobre eutanasia, no tomando en cuenta su grado de instrucción, lo que es de preocupar debido a que, en la actualidad, con el auge tecnológico, la población general adquiere mayores conocimientos sobre la eutanasia y los distintos términos relacionados con ella. ${ }^{17}$ De aquí podemos inferir que, si la población profesional médica no mejora su grado de conocimiento, no podrá existir una comunicación ni entendimiento adecuado con la población general.

En un estudio realizado en México entre estudiantes de medicina en el 2006, encontraron que el 79\% estaba en contra de la eutanasia en pacientes en estado de coma irreversible, y en otra pregunta el 56\% consideró que la eutanasia provocará una devaluación de la profesión médica. ${ }^{18} \mathrm{~A}$ partir de los resultados encontrados en el presente trabajo, se demostró que una tercera parte se encuentra totalmente de acuerdo con la aceptación de la eutanasia en pacientes en situaciones críticas y un tercio simplemente de acuerdo. Aproximadamente dos terceras partes de la muestra está totalmente en desacuerdo respecto de la eutanasia y la considera como desvalorización de la profesión médica.

En el 2009, en un estudio en Brasil, se interrogó a 30 médicos especialistas sobre este tema. De ellos, sólo el 63.3\% conocía la definición de la eutanasia, lo que nos permite entender que el grado de instrucción no es concluyente para determinar los conocimientos sobre este tema. ${ }^{19}$ Mínimas son las diferencias de respuestas entre los estudiantes y profesionales de medicina en este estudio, 
obteniendo resultados por encima de las tres cuartas partes en los tres grupos estudiados.

Quintana O., en el 2003, comenta respecto de la consideración ética que éste es un tópico que enfrenta distintos entes, no sólo en el ámbito de la salud, sino en toda la sociedad, lo que genera discusiones y a veces conflictos dentro de la misma. Si bien en la actualidad la eutanasia no es el epicentro de las discusiones, tarde o temprano llegará a serlo y la comunidad médica, junto con las escuelas de medicina, deberán estar preparadas para afrontar esta cuestión de principios, ${ }^{20}$ por lo que en este trabajo se evidencia una muy buena respuesta en cuanto a la consideración ética de la eutanasia, presentándose en la población con niveles medios de conocimiento en más de la mitad de los estudiados, mientras que en personas con niveles altos de conocimientos se observó una baja proporción.

De los individuos que apoyaron la eutanasia, más de la mitad fueron mujeres, con un resultado estadísticamente significativo, lo que, en contraste con el estudio realizado por Ramírez Rivera et al. en Puerto Rico, cuya mayoría de participantes que estaban dispuestos a practicar la eutanasia eran del género masculino. ${ }^{21}$ Según Bastos Brandalise V. et al., en su estudio realizado en Brasil, el número de participantes que recibieron la solicitud de ayuda por parte de un paciente con enfermedad terminal para acelerar su proceso de muerte fue del 11\%, mientras que el 89\% nunca recibió la solicitud. En cambio, en el mismo estudio respondió afirmativamente un $20.3 \%$, que meditó sobre la idea de ofrecer ayuda ante la solicitud de acabar el sufrimiento de algún paciente acelerando su muerte. En la investigación actual se observó la influencia del contacto con un paciente con enfermedad terminal y su relación con el apoyo a la eutanasia, demostrando una influencia significativa. ${ }^{22}$

Con respecto a la situación legal en Venezuela, en el Código de Deontología Médica del año 2004, se presentan ciertas deficiencias en lo que respecta al capítulo referente al enfermo terminal. Se demuestra así que dicho código se tornó más limitante en compara- 
ción con el código del año 1985, donde implícitamente el paciente tiene menos libertad de solicitar cómo desea sobrellevar o terminar su sufrimiento y más limitaciones al médico tratante. ${ }^{23}$

En el código deontológico del año 1985, el artículo 75 habla de que no sólo el enfermo debe ser atendido por profesionales competentes y con actitudes positivas en la aplicación de tratamientos en el área de enfermos incurables, sino que, además, no debe sufrir prejuicios en relación con la muerte, mientras que en el código deontológico del año 2004, en el artículo 80, se reitera la importancia de no tener prejuicios en relación con la muerte y se incorpora la paciencia y los tratamientos paliativos por un equipo interdisciplinario, manteniendo el cuidado del enfermo terminal hasta sus últimas instancias. Hay que destacar que esto último hace referencia a lo que podría ser la ortotanasia.

Por otra parte, el artículo 82 indica que el enfermo terminal no debe ser sometido a la aplicación de medidas de soporte vital derivadas de la tecnología, las cuales sólo servirán para prolongar la agonía, haciendo referencia implícitamente a la distanasia, y cuando posteriormente se hace referencia a ésta, se debería destacar que no se toman en cuenta definiciones como ortotanasia ni eutanasia, ya que son parte del contexto. Por otro lado, el artículo 84 «obliga» al médico al alivio del sufrimiento humano pero, al mismo tiempo, no puede provocar deliberadamente la muerte del enfermo, aun cuando éste o sus familiares lo solicitasen, creando una disyuntiva entre quienes son los que realmente deciden y actúan sobre la calidad de vida del paciente.

\section{Conclusiones}

Los resultados demostraron que existe conocimiento acerca de la eutanasia; en la población estudiantil en particular se reveló el porcentaje más alto. Es un tema que invitó a la reflexión de los partici- 
pantes mientras respondieron la encuesta. No obstante, el nivel de conocimiento del tema de toda la muestra es medio, hecho que invita a profundizar en este aspecto, pues la eutanasia es una posibilidad dentro de la terapéutica médica, para la cual el profesional de la medicina debe estar preparado.

En la formación académica del médico, la eutanasia es un contenido de la materia de bioética; no obstante, es un tema que se limita a la conceptualización del término y su estatus legal en el país de estudio, pero es necesario profundizar en el mismo debido a que tiene implicaciones varias; por ejemplo, filosóficas, sociológicas y, por supuesto, legales, por mencionar algunas áreas del conocimiento con las que se interrelaciona.

Vale destacar que la eutanasia no fue percibida de manera negativa por la muestra estudiada, demostrando una alta significancia en atención a las implicaciones éticas que rodean este concepto, tanto en la sociedad como en la profesión médica. Anteriormente, era un tema tabú o era obviado para evitar confrontar posiciones éticas y morales ante la vida, siendo incluso en la actualidad un tema de conocimiento del público general.

Tomando en cuenta el marco legal existente, pareciera que existe una probable descontextualización referente al tema, porque no hay actualización de términos referente a la vida y la muerte, aun cuando la sociedad hoy día (15 años después de la última actualización del Código Médico Deontológico) es otra. Esto pudiese ser consecuencia de la explosión del progreso técnico y científico, que ha llevado a la modificación del pensamiento respecto de la muerte, aceptando que no es ajena a la vida, siendo así un tema de discusión social.

En la formación del médico, los Comités de Bioética de las distintas universidades y hospitales debieran realizar charlas o cursos acerca del tema con mayor frecuencia, así como en la unidad curricular de bioética se debe ampliar el tema acerca de la eutanasia y sus términos asociados, como son la distanasia y la ortotanasia, así como también se recomiendan modificaciones legales con respec- 
to a los códigos médico-deontológicos o a cualquier ley que lo amerite, de acuerdo con las leyes de cada país, teniendo en cuenta un apartado que tipifique de manera explícita los conceptos respecto al manejo de la muerte, los cuales son eutanasia, distanasia, ortotanasia y suicidio asistido.

\section{Notas bibliográficas}

1 Cuervo Pinna, M. A., Rubio, M., Altisent Trota, R., Rocafort Gil, J., et al. Investigación cualitativa sobre el concepto de eutanasia, entre médicos españoles. Revista de Calidad Asistencial; 2016; 31(1): 18-26. https://doi.org/10.1016/j. cali.2015.07.002

${ }^{2}$ Martínez, I., Eutanasia. En: De Mestral, E. Manual de Bioética. Asunción: EfaCIM; 2014; p. 173-182.

${ }^{3}$ Álvarez del Río, A. El derecho a decidir: eutanasia y suicidio asistido. Cir. Gen.; 2013; 35 (Supl. 2): S115-S118. Recuperado en: http://www.medigraphic.com /cirujanogeneral

${ }^{4}$ Rodríguez-Domínguez, Z., Casado-Méndez, P., Méndez-Jiménez, O., CasadoTAMAYO, P., FerReR-MAgadÁN, C. Percepción familiar del enfermo terminal sobre la distanasia y ortotanasia en la Atención Primaria. MULTIMEd Revista Médica Granma; 2017; 19(1). Recuperado en: http://www.revmultimed.sld.cu/index.php/ $\mathrm{mtm} /$ article/view/499 https://doi.org/10.1016/s1134-2072(12)70398-5

${ }^{5}$ Ríos González, C. M., De Benedictis Serrano, G. A., Contreras lugo, L. V., Córdova Rivas, G. J. ET AL. Análisis bibliométrico sobre las publicaciones de eutanasia en Scopus, MEDLINE/Pubmed, Science Citation Index y Scielo. Rev. Med. Hered. 2018; 29: 125-126. Recuperado en: https://doi.org/10.20453/rmh.v 29i2.3355

${ }^{6}$ Carrasco, M., Víctor, Hugo, Crispi, Francisca. Eutanasia en Chile: una discusión pendiente. Rev. Méd. Chile; 2016; 144(12): 1598-1604. Recuperado en: https://doi.org/10.4067/S0034-98872016001200012

${ }^{7}$ Nombela, C., López, F., Serrano, J. M., Postigo, E., Abellán, J., Prensa, L. La eutanasia: perspectiva ética, jurídica y médica. Debate actual 2009; 10: 56-75. Recuperado en: https://eprints.ucm.es/11693/1/La_Eutanasia_perspectiva_etica_ juridica_y_medica.pdf

${ }^{8}$ ORganizAción DE NAcIONES UnIDAS. La Declaración Universal de Derechos Humanos. París: ONU; 1948. https://doi.org/10.2307/j.ctv86dh99.12

${ }^{9}$ Venezuela. Código Médico Deontológico. Aprobado durante la CXL reunión extraordinaria de la Asamblea de la Federación Médica Venezolana. Cumaná: Federación Médica Venezolana, 24-26 de octubre 2004. https://doi.org/10.1017/ s0250569x00017416 
Conocimiento de la eutanasia en estudiantes y profesionales de medicina

10 TANNEHILL, W. Sketches of the History of Literature: From the Earliest Period to the Revival of Letters in the Fifteenth Century. BiblioLife, editor; 2016; pp. 98-100. ${ }_{11}$ RoJAS, K. R. Nivel de conocimientos y actitudes acerca de la eutanasia en internos y médicos residentes de cinco hospitales del departamento de Lambayeque en el 2015. RegistNacTrablnvestig; Perú, 2017. https://doi.org/10.1016/j.acu.2017. 09.002

${ }^{12}$ AnNeser, J., Jox, R., Borasio, G. D. Physician-assisted suicide, euthanasia and palliative sedation: attitudes and knowledge of medical students. GMS J. MedEduc. 2016; 33(1): 1-14. Recuperado en: https://doi.org/10.3205/zma001010

${ }^{13}$ MARTíneZ, A., Ríos, F. Los conceptos de conocimiento, epistemología y paradigma, como base diferencial en la orientación metodológica del trabajo de grado. Revista Cinta de Moebio; 2006; 25: 111-121. Recuperado en: https://revistas.uchi le.cl/index.php/CDM/article/view/25960/27273 https://doi.org/10.4067/s0717-554x 2009000100004

${ }^{14}$ VARgas Melgarejo, L. Sobre el concepto de percepción. Alteridades; 1994; 4 (8): 47-53. Recuperado en: https://www.redalyc.org/articulo.oa?id=74711353004

${ }^{15}$ OVIEDO, G. La definición del concepto de percepción en psicología con base en la teoría Gestalt. Revista de Estudios Sociales; 2004; 18: 89-96. Recuperado en: http://www.scielo.org.co/pdf/res/n18/n18a10.pdf https://doi.org/10.7440/res18.20 04.08

${ }^{16}$ Ríos González, C. M., De Benedictis Serrano, G. A., Córdova Rivas, G. J., Contreras Romero, M. L., Contreras Lugo, L. V. Conocimientos y percepción sobre eutanasia en estudiantes de medicina de diecisiete países latinoamericanos, 2017. Mem. Inst. Investig. Cienc. Salud. 2018; 16(3): 58-65. Recuperado en: https://doi.org/10.18004/mem.iics/1812-9528/2018.016(03)58-065

${ }^{17}$ Pichardo García, L. M. G. ET AL. Conceptos relacionados con el final de la vida. Gac. Med. Mex. 2019; 155: 149-155.

${ }^{18}$ Casas, M. de la L., Pichardo, L. M., Manzano M. del C., Torres, J. C., Gómez SANCHEZ, J. Encuesta piloto comparativa de opinión de médicos, enfermeras y estudiantes de medicina sobre el suicidio asistido en un hospital privado del Distrito Federal. Pers. y Bioética 2009; 11(2): 186-95. Recuperado en: http://www.scielo. org.co/pdf/pebi/v11n2/v11n2a08.pdf https://doi.org/10.1016/s0212-69 82(09)000 08-1

19 Pricoli Vilela, L., Caramelli, P. Knowledge of the Definition of Euthanasia: Study With Doctors And Caregivers Of Alzheimer's Disease Patients. Rev. Assoc. Med. Bras.; 2009; 55 (3): 263-7. Recuperado en: https://doi.org/10.1590/s0104-42 302009000300016

${ }^{20}$ QuINTANA, O. Los objetivos de la medicina. RevCalidAsist; 2003; 18(2): 132-135.

21 RamiRez Rivera, J., Cruz, J., Jaume Anselmi, F. Euthanasia, assisted suicide and end-oflife care: attitudes of students, residents and attending physicians. Pr. Health Sci. J. 2006; 25(4): 325-329. Recuperado en: http://prhsj.rcm.upr.edu/ index.php/prhsj/article/view/283/173

22 Bastos Brandalise, V., Pertile Remor, A., De Carvalho, D., luiz Bonamigo, E. Suicidio asistido y eutanasia en la perspectiva de profesionales y estudiantes 
R. D’Amico López, G. Benedictis Serrano, H. Coiran Mendoza, J. Castro Álvarez

de un hospital universitario. Rev. Bioét. 2018; 26 (2): 217-27. Recuperado en: https://doi.org/10.1590/1983-80422018262242

${ }^{23}$ Venezuela. Código Médico Deontológico. Aprobada durante la LXXVI reunión extraordinaria de la Asamblea de la Federación Médica Venezolana. Caracas: Federación Médica Venezolana, 20 de marzo de 1985. https://doi.org/10.18273/ revbol.v41n1-2019006 